\title{
FIGO Stage IVB Ovarian Cancer
}

National Cancer Institute

\section{Source}

National Cancer Institute. FIGO Stage IVB Ovarian Cancer. NCI Thesaurus. Code

C128095.

Ovarian cancer with hepatic and/or splenic parenchymal metastasis, metastasis to extraabdominal organs (including inguinal lymph nodes and lymph nodes outside of the abdominal cavity). (FIGO, 2014) 